\title{
Civilisations
}

Revue internationale d'anthropologie et de sciences

humaines

$50 \mid 2002$

Itinéraires belges aux Amériques

\section{Le « Métissage » et la culture " populaire » au Brésil}

Ethnologie de la fête du Bumba Boi

Didier de Laveleye

\section{(2) OpenEdition}

Journals

Édition électronique

URL : http://journals.openedition.org/civilisations/3404

DOI : $10.4000 /$ civilisations.3404

ISSN : 2032-0442

Éditeur

Institut de sociologie de l'Université Libre de Bruxelles

\section{Édition imprimée}

Date de publication : 1 décembre 2002

Pagination : 153-174

ISBN : 2-87263-180-1

ISSN : 0009-8140

Référence électronique

Didier de Laveleye, «Le « Métissage » et la culture « populaire » au Brésil », Civilisations [En ligne],

50 | 2002, mis en ligne le 01 décembre 2004, consulté le 30 avril 2019. URL : http://

journals.openedition.org/civilisations/3404; DOI : 10.4000/civilisations.3404

(c) Tous droits réservés 


\title{
LE « METISSAGE » ET \\ LA CULTURE « POPULAIRE » AU BRESIL ETHNOLOGIE DE LA FETE DU BUMBA BOI
}

\author{
DIDIER DE LAVELEYE
}

\begin{abstract}
"Pour qu'il y ait identité, société, culture ou ethnie, il n'est pas nécessaire que les agents se mettent d'accord sur ce qui définit cette culture: il suffit qu'ils s'entendent pour débattre ou négocier sur les termes de l'identité, sur ce qui la fonde comme problème " (Amselle 1990: 65).
\end{abstract}

Depuis de nombreuses années, une "révolution scientifique " ne cesse de bouleverser le domaine des études culturelles en général et celui de l'américanisme en particulier. Ce mouvement, qui n'est pas unitaire bien qu'il englobe autant l'anthropologie que l'histoire, consiste à reposer la question du découpage culturel de l'humanité. Je ne me risquerai pas, à ce stade du développement historique des sciences de la culture, à tenter de brosser un tableau général des débats, querelles et remises en doutes qui animent de toutes parts nos disciplines et qui, jusqu'à ce jour, parviennent uniquement à les diviser plus encore. Mais si l'absence de recul historique ne nous permet pas encore d'en envisager l'issue, cette phase critique - et combien passionnante nous accorde d'une certaine manière la liberté du parti pris.

Le bouleversement que suscite la remise en question du découpage culturel de l'humanité ne signifie pas nécessairement que l'on remette en doute la pertinence d'une certaine "spécificité culturelle" (formes de comportements, mode de pensée, etc.) propre à chaque collectivité, groupe, ethnie ou civilisation. Il ne s'agit pas de faire table rase de la notion de culture mais plus positivement, de réévaluer les fondements paradigmatiques des découpages culturels. Ces découpages étaient classiquement conçus comme constituant des ensembles déterminés (les "cultures"), composés d'éléments essentiels (les " traits culturels "), cohérents, homogènes et parfaitement intégrés dans chaque ensemble; c'est-à-dire organisés par une «logique " interne (de type fonctionnelle, structurale, systémique, etc) qui conduirait à naturellement perpétuer le modèle traditionnel (sauf grave conflit, guerres, etc). Trois critiques 
fondamentales ont pu être apportées à cette formulation schématique de la culture :

1.- A de trop rare exception près, il n'existe pas d'isolats culturels. Dans la plupart des cas le fait de décrire une société et d'expliquer son fonctionnement comme si elle était seule dans son paysage, relève d'une fiction inavoué qui vise à occulter ou à minimiser l'importance des contacts, des échanges ou des guerres interethniques; et à sous-estimer les caractéristiques de la périphérie des ensembles centralisés, le rôle de certains passeurs culturels, de même que l'aspect arbitraire, poreux et instable des frontières.

2.- La culture n'est pas nécessairement un tout ordonné, tel un système qui posséderait une sorte de stabilité fondamentale vers laquelle elle serait perpétuellement attirée, sauf accident historique imprévisible qui induirait une rupture de ce mystérieux équilibre. Plutôt que de postuler l'intégration et l'homogénéité des idées et des pratiques dans chaque espace culturel, il convient de l'expliquer ou mieux, d'évaluer les mécanismes qui induisent ou infléchissent cette homogénéité.

3.- L'identité culturelle ou ethnique d'un groupe n'a d'autre réalité scientifique que ce que les acteurs en disent ou en font. Aussi, cette construction sociale et historique de l' « ethnie » doit être radicalement distinguée de la catégorie analytique qui permet à l'anthropologue d'appréhender en un seul terme un ensemble social bien souvent instable, hétérogène et mouvant.

La plupart des chercheurs en sciences sociales s'entendent sur le bien fondé de ces trois critiques, ce dont témoigne l'abondance des thèmes actuels qui touchent directement ou indirectement au paradigme du découpage culturel : identités, relations interethniques, frontières, métissage, syncrétisme, historicité et invention de la tradition, etc. Mais il est cependant difficile d'identifier aujourd'hui un début de consensus sur l'importance à accorder à ces critiques. Pour beaucoup de chercheurs encore, les dimensions hétérogènes et instables de nos ensembles culturels classiques sont certes des questions méritoires et utiles, mais restent relativement secondaires par rapport aux éléments stables et totalisants qui nous permettraient d'organiser la plupart de nos données et observations ethnographiques. La problématique du découpage culturel ne serait alors pleinement posée que dans le contexte spécifique de la fusion ou de l'assimilation de civilisations distinctes qui, en Amérique, aurait débuté par la Conquête et se poursuivrait aujourd'hui par la «mondialisation ». A l'opposé de cette conception classique -mais néanmoins problématiqued'une répartition de l'humanité entre "traditions" et «modernité », d'autres chercheurs (dont je suis) reposent au contraire le postulat de l'unité de l'homme en affirmant que les phénomènes de métissage, d'hybridation et de découpage culturel, participent de toutes les cultures à tous les instants de leur histoire.

Cette position est sans doute celle de Jean-Loup Amselle, pour qui "l'erreur des anthropologues culturalistes consiste à ne pas traiter la culture comme une solution instable dont la perpétuation est par essence aléatoire " (Amselle 1990:56). Dans cette perspective, il conviendrait d'observer une « culture » 
non plus exclusivement sous ses aspects homogènes, stables et cohérents, ni à l'inverse exclusivement sous l'angle d'un mélange chaotique ou complexe déjà élaboré, mais plutôt de saisir empiriquement les éléments qui permettent -ou pas- à un groupe humain de "débattre ou négocier sur les termes de (son) identité, sur ce qui la fonde comme problème» (Amselle 1990:65). Ainsi, en systématisant quelque peu les propos de l'anthropologue africaniste, on peut donc considérer que le "découpage culturel " n'est pas un pré-requis de l'humanité, il n'est pas binaire (absent/présent), mais il constitue au contraire le moteur de chaque "culture". Dans cette perspective, les mécanismes qui accompagnent cette tendance au découpage culturel, peuvent devenir à eux seuls un objet d'observation et d'analyse. Toute société, pour se former, mais aussi pour évoluer et se perpétuer, chercherait à négocier son découpage au sein d'un espace social plus large, diversifié et hétérogène. Mais en aucun cas cette négociation ne pourra produire un accord parfaitement uniforme et immuable : l'illusion de l'immuabilité est un phénomène sociologique courant qui n'entretient cependant aucun lien de détermination avec la réalité historique d'une reproduction à l'identique. Même si les "Traditionnels " postulent unanimement que leurs actions sont conformes à celles de leurs ancêtres et que les «Modernes » pensent au contraire s'en distinguer -ceci indépendamment de la réalité historique de telles assertions- l'homogénéité culturelle, c'est-à-dire la cohérence, la stabilité et l'intégration de leurs modes de faire et de penser, relève ici comme ailleurs d'un enjeu politique qui doit sans cesse être alimenté ou reconstruit.

L'Amérique constitue sans doute un terrain privilégié pour l'étude de ces phénomènes de découpage culturel. Au niveau des nations, ces phénomènes posent d'abord la question de l'identité d'ensembles multiculturels. Le découpage est alors sans doute mis au service d'une homogénéisation d'un espace politique commun, mais aussi au service d'une distanciation ou d'une reformulation de ses références culturelles d'origine, tant vis-à-vis des indigènes que des anciennes métropoles. L'évolution des termes et des dimensions de l'espace social (devenu) national induit donc également un redécoupage des unités culturelles qui sont englobées dans l'espace de relation interethnique. Cela peut conduire à une cristallisation des identités et à une ségrégation des groupes, tout comme à l'abolition des frontières de l'endogamie et à la valorisation du métissage. Aussi, certaines unités de l'espace social élargi préserveront leur label ethnique comme critère d'identification et de pérennisation, indépendamment des phénomènes de fission ou de fusion qui auront quelquefois été nécessaires pour entretenir une conscience d'appartenance légitime à une "ethnie ". D'autres individus abandonneront par contre toute référence à un label ethnique autre que celui de la nation. Mais l'absence de label ethnique propre n'empêche pas les individus d'établir des relations sociales spécifiques et récurrentes qui deviennent donc elles-mêmes l'objet d'unités sociales discrètes qui, à leur tour, auront tendances à cerner les contours de leur spécificité, de leur «culture». C'est ce phénomène d'appréhension d'une culture par des groupes métis, que nous allons maintenant observer et analyser dans son contexte brésilien. 


\section{LE METISSAGE AU CCEUR DU MYTHE NATIONAL BRESILIEN}

Comme l'a parfaitement démontré l'anthropologue brésilien Roberto DaMatta, le Brésil est une nation multiraciale qui, à l'opposé du modèle nordaméricain, cherche à faire l'éloge du métissage plutôt que celui d'une égalité raciale (DaMatta 1987:58-85). Aux Etats-Unis, la ségrégation raciale est au fondement du modèle égalitaire, selon l'ambiguité de la formule " tous égaux, mais différents ». Dans ce contexte, le moindre indice biologique suffit à répartir les individus en différents groupes raciaux, sans qu'aucune place ne soit faite au métis : on est soit Blanc, soit Noir, ou encore Irlandais, Latino, Chinois, etc. Au Brésil, par contre, l'absence de ségrégation raciale stricto sensu laisse plutôt la place à une conception hautement hiérarchisée et profondément inégalitaire de la société, à la tête de laquelle se trouve le Blanc, tandis que le Noir et l'Indien en sont les deux piliers. Aussi, dans ce pays, on ne sait jamais trop bien si ce sont les critères raciaux qui déterminent l'appartenance à une classe sociale ou l'inverse. Ainsi, quelqu'un qui a la peau apparemment très noire et qui possède le pouvoir économique ou politique est souvent traité de moreno (Blanc à la peau noircie au soleil) plutôt que de Noir (preto) ou de Nègre (Negro). Dans ce pays, les labels raciaux définissent moins des groupes discriminés les uns des autres que les stéréotypes attachés à chaque couche sociale, tels que l'intelligence et le pouvoir d'entreprise de la classe des Blancs, la force physique et le tempérament festif des Noirs, l'esprit guerrier et proche de la nature des Indiens. Mais au Brésil, personne ne revendique une pureté raciale. Même parmi les couches les plus huppées, le Brésilien est toujours fier de revendiquer au moins une goutte de sang d'un lointain ancêtre Noir et/ou Indien. Le métissage est à ce titre considéré comme le ciment de la nation brésilienne, sa «valeur ajoutée " en quelque sorte, comparativement aux nations européennes.

On comprend dans ce contexte la difficulté des mouvements identitaires indigènes et afro-brésiliens à émerger comme force politique. Malgré leur soutien par des organismes nord-américains, leur principal problème est que le seul critère racial -imposé à l'origine par la société coloniale- n'est pas suffisant pour susciter l'adhésion de leurs membres potentiels. Le modèle ségrégationniste nord-américain, même s'il est objectivement mis ici au service d'une lutte contre une injustice sociale, s'applique très mal dans un pays qui n'hésite pas à valoriser son métissage. Pourtant, le métissage n'est pas le vecteur d'une identité spécifique à l'intérieur du Brésil. Le terme mestiço (métis) n'est jamais utilisé pour désigner quelqu'un en particulier, dans la mesure où ce critère est idéologiquement partagé par tous. En outre, les termes plus directement liés à une appréciation de type raciologique (Branco, Negro et Indio) sont habituellement évités au quotidien ou sont utilisés pour désigner des individus non apparentés aux personnes présentes. Les termes désignant les nuances entre les catégories raciales sont par contre couramment employés, tels que moreno qui désigne un Blanc à la peau foncée, preto pour indiquer la couleur noire de la peau plutôt que la race proprement dite (Negro), et Caboclo qui caractérise à l'origine la peau cuivrée de l'Indien. Ces termes nuancés sont préférés sans doute parce qu'ils échappent relativement aux jugements des stéréotypes habituels: intuitivement, le 
Brésilien considère plus correcte de parler d'un Indien vêtu à la brésilienne comme d'un Caboclo. De même, le terme moreno convient autant au Noir huppé qu'au Blanc basané. Enfin, on est plus facilement riche et preto que riche et Negro. Aussi, ces catégories raciologiques atténuées ne relèvent pas d'une volonté de formaliser les interstices du métissage brésilien bien qu'ils alimentent implicitement les préjugés raciaux. Ces termes sont en réalité extrêmement flottants puisque leur emploi dépend tout à la fois du contexte, de l'appréciation subjective de chaque locuteur sur la couleur de sa propre peau, tout comme sur celle de son interlocuteur et du public en présence.

Cette flexibilité manifeste de la langue et des critères d'identification des personnes ou des groupes, empêchent très certainement l'édification d'une conscience de soi qui se distingue du sentiment diffus d'un nationalisme brésilien. Le racisme à la brésilienne empêche donc l'émergence d'identités autonomes puisque tout le monde est finalement métis -et donc Brésilien. Mais l'identité brésilienne ne relève pas d'une conception égalitaire des citoyens puisque pour être Brésilien -et donc métis- il faut être positionné au sein d'une structure hiérarchisée et polarisée entre les trois références racıales, le Branco, le Negro et l'Indio.

Il résulte de tout ceci, que quand les spécialistes de la culture brésilienne traitent de manifestations et de comportements spécifiques à un groupe ou à une région du pays, et que ceux-ci ne peuvent être rangés sous les labels $d^{\prime}$ "africain », $d$ ' " européen » ou d' "amérindien», alors ils n'ont d'autres choix semble-t-il, que de désigner ces éléments à l'aide du terme «populaire ». L'idée de «populaire » dénote indubitablement une notion de classe sociale, ce qui ne manque pas d'évoquer une fois encore une conception hiérarchisée des cultures en présence. De ce fait, caractériser un catholicisme ou un folklore de «populaire » ou bien, dans son contexte rural, de "paysan » (camponês), alimente le racisme à la brésilienne qui consiste à s'approprier le métissage et à réduire la diversité ethnique et culturelle du pays à des relations sociales préétablies et hiérarchiquement figées. L'anthropologue est-il pour autant condamné à souscrire à cette représentation de l'espace social brésilien au point de ne pouvoir distinguer d'autres découpages que celui d'une stratification sociale balisée de préjugés raciaux?

A l'aide d'un exemple ethnographique particulier -celui de la fête du Bumba Boi-, je voudrais illustrer de quelle manière des groupes "populaires" (donc métis) s'approprient cette représentation "officielle » de la nation brésilienne. Nous verrons qu'à l'aide d'une transposition de cette représentation sur une scène de théâtre et de danse, les groupes " populaires " parviennent à s'approprier les termes de leur identité métisse pour finalement, commencer à écrire une autre histoire.

\section{DU THEATRE DES RACES A L'EMERGENCE DU METIS : LE BUMBA BOI}

Le Bumba Boi est, selon la terminologie des folkloristes brésiliens, un auto popular, un "théâtre populaire », associant tout à la fois la comédie, la musique et la danse, mais aussi le burlesque, le lyrisme, la moquerie et la prière. 
Ces festivités sont réalisées par des troupes aux allures folkloriques, composées de musiciens et de danseurs richement costumés qui se rassemblent autours d'un masque représentant un bœuf, le Boi. Ce masque, construit à partir d'une structure en rotin d'une envergure de 1,5 m environ est décoré de milliers de perles multicolores et est porté par un danseur, le miolo do boi, qui se dissimule sous une robe. Dans sa version canonique, outre le bœuf, la comédie réunit généralement trois groupes de personnages : les vachers (vaqueiros), dirigés par un maître (fazendeiro ou dono) et son intendant (feitor) ; les bouffons (palhaços), composés le plus souvent d'un ou deux couples de "pauvres noirs"; et enfin les Indiens (indios ou caboclo de pena). Le groupe des vachers est sans conteste le plus richement vêtu d'habits ornés de perles, de chapeaux et de rubans multicolores. La bande d'Indiens est systématiquement parée de plumes, tandis que les bouffons, eux, ne possèdent pas de vêtement caractéristique hormis l'un ou l'autre détail suffisant à évoquer la caricature du vagabond ridicule.

Avant d'entamer une analyse du jeu théâtral proprement dit et d'isoler l'intrigue qui est censée animer les différents personnages de cette comédie, il convient de préciser que celle-ci est en réalité très peu jouée. Les troupes de Bumba Boi préfèrent aujourd'hui réaliser des représentations publiques consistant uniquement à danser et à chanter (mais avec les costumes de comédie). Les chants eux-mêmes ne font plus que très rarement référence à la comédie "traditionnelle" pour composer davantage sur un répertoire tantôt purement lyrique et poétique, tantôt simplement comique et satirique. Lors de ces manifestations publiques, le chanteur principal commence par entonner une strophe avant d'être rapidement accompagné (et couvert) par le groupe des percussionnistes qui se tient ramassé derrière lui. La chorégraphie n'est généralement pas très développée. Elle consiste à former des rondes, au centre desquels virevolte le masque du bœuf, en piétinant le sol d'avant en arrière au rythme de larges tambours bi-membranes (les zabumbas) ou de grands tambourins (pandeiros) et de claquettes de bois (matracas), tout en entraînant une foule de spectateurs qui danse avec et autour des personnages costumés.

A certaines occasions, les troupes déambulent dans les rues sous forme d'une procession. Dans ce cas, les danseurs costumés prennent la tête du cortège, tout en avançant au pas de danse, tandis que le bœuf court et voltige au milieu. Immédiatement derrière la troupe des comédiens costumés suivent les joueurs de tambour ou de tambourin. Enfin, en queue de file, une quantité innombrable de joueurs de matracas, souvent improvisés et non costumés, accompagnent le rythme général en claquant frénétiquement l'un contre l'autre leur deux morceaux de bois. Les jours chauds de cette fête, aux alentours de la Saint Jean, le 24 juin, ces joueurs de matracas (qui sont en fait composés d'une majorité de simples sympathisants ou de passionnés du moment) peuvent atteindre plusieurs centaines d'individus ramassés derrière un seul masque. On imagine qu'à cette hauteur du cortège, le chanteur et les danseurs se trouvant à quelques dizaines de mètres de là, seule l'ardeur du rythme et le brouhaha des claquements peuvent être entendus et soutenir la foule endiablée. Aussi, ni le lyrisme du chant supposé diriger la partition musicale, ni les rôles des acteurs de comédie qui ouvrent la marche, ne peuvent suffire à expliquer pareil emballement collectif. 
Sans doute qu'une part du succès du Carnaval dans d'autres régions du Brésil, tient au même facteur d'emballement d'une foule ramassée autour d'un même rythme simple et répétitif. L'importance d'une fête, du point de vue de sa manifestation collective, ne peut certainement pas se mesurer à ses seuls éléments signifiants. Cependant, dans la région du Maranhão, le Carnaval est quant à lui considéré comme en phase de décadence, à l'inverse du Bumba Boi dont on dit souvent qu'il est la fierté de ses habitants. Aussi, la différence d'engouement entre le Carnaval (considéré comme décadent) et le Bumba Boi (vécu comme en plein essor), qui s'animent pourtant tous deux à l'aide d'un même type de musique rythmique, ne peut trouver sur cette base de réponse satisfaisante. II convient dès lors de distinguer -sans pour autant complètement les dissocicr- les éléments signifiants de cette fête (ses représentations et symboles) des mécanismes collectifs qu'elle induit sur sa population (forme et intensité de l'engouement).

\section{L'INTRIGUE DU BCEUF}

Aveuglé sans doute par ce que j'en avais lu et aussi par ce qu'on m'en raconta lors de ma première découverte de cette fête en ville, à São Luis, j'ai longtemps pensé que la signification de cet amusement populaire était à chercher dans l'intrigue de la comédie du Bœuf. Or ma première surprise fut de découvrir que l'action théâtrale qui est quelquefois (mais rarement) jouée en ville et que beaucoup croient semblable d'année en année (avec cependant quelques variantes), n'est pas la version que les paysans jouent pour eux dans leurs villages. Il existe effectivement une version « officielle» de cette comédie du bœuf, que les écrits des folkloristes considèrent comme " traditionnelle » ou canonique, et qui est effectivement à peu près identique quelle que soit la troupe qui l'interprète mais qui n'est jouée que sur commande, généralement pour le compte d'un riche propriétaire terrien (dono) ou celui d'une municipalité ${ }^{1}$.

Cette version «traditionnelle » du Bumba Boi, celle que tout le monde connaît et que la bourgeoisie de la capitale croit unique et semblable partout, est une satire comique d'une exploitation coloniale d'élevage, une fazenda, dans laquelle on attribue littéralement le "beau rôle " à ses propriétaires et celui du bouffon ridicule aux pauvres paysans. Au centre de l'action burlesque se trouve le plus beau bœuf de l'exploitation (le boi, qui est en fait un taureau reproducteur et non pas un animal castré), fierté de son propriétaire, le dono ou fazendeiro, qui est bien sûr un Blanc. A côté de ce dernier, son intendant (mandante, feitor ou capataz) joue le rôle de narrateur ou plutôt ici, de chanteur principal du spectacle; tandis que sous ses ordres, les vachers (vaqueiros) forment une troupe de danseurs joliment vêtus de chapeaux, de perles et de rubans. A distance de ce groupe d'employés de la fazenda, un couple de pauvres agriculteurs, vivant en marge du domaine, jouent les bouffons ridicules, paresseux et maladroits. L'identité « nègre » (negro) de ce couple est quelquefois appuyée d'un masque ou, si nécessaire, d'un barbouillage des visages à la cendre. L'épouse est enceinte. La comédie commence lorsque celle-ci exprime le 
désir de manger la langue (ou le foie) du bœuf et supplie son homme de lui en rapporter un morceau. L'homme, criant sa misère et effrayé par la menace de perdre son futur enfant, finit par céder et capture le bel animal afin de lui prendre un morceau de sa viande. Constatant la disparition de son bœuf (taureau) préféré, le fazendeiro envoie ses vachers à sa recherche. L'animal est découvert mourrant. Le couple de voleurs s'enfuit. On envoie alors chercher les Indiens, parés de leurs plumes (caboclos de pena ou tapeiros), afin que ceux-ci retrouvent la trace des responsables. Le couple de voleurs est finalement rattrapé. Heureusement, parmi les Indiens, le doutor do mato ou pajé (chamane local) guéri l'animal blessé, de sorte que la comédie se termine dans la fête et la joie collective ${ }^{2}$.

Bien que l'époque à laquelle se réfère cette comédie "traditionnelle » n'est pas explicite, plusieurs éléments indiquent qu'on doit la situer proche de l'époque de l'esclavage. L'élément le plus frappant est l'appel d'Indiens pour rattraper les malfaiteurs. Maria de Carvalho a relevé une variante de cette version où ces Indiens demandent à être baptisé avant d'entrer au service du fazendeiro (Carvalho 1995:181-184). Durant l'esclavage, il était effectivement courant de louer les services $d$ 'indigènes, parfaits connaisseurs de la région et experts réputés dans le pistage de leurs ennemis en forêt, afin de traquer les esclaves fugitifs. Cependant, ce sont rarement des groupes entiers qui étaient contractés mais plus couramment des individus isolés, souvent des métis (et sans doute déjà baptisés), qui accompagnaient et guidaient des troupes de l'armée régulière. Suite à l'augmentation des fuites et révoltes d'esclaves africains, on instaura dans la région à partir de la seconde moitié du XIX" siècle, des " capitão do mato " (capitaines de brousse), spécialement destinés à la capture des Nègres fugitifs.

D'autres éléments rapportés dans les écrits des folkloristes, comme la totale soumission et la crainte des vachers vis-à-vis de leur maître ou quelquefois aussi les tortures physiques (castigos) que reçoivent les voleurs après leur capture, puisent sans doute leur inspiration dans cette époque coloniale et esclavagiste. Tout indique donc que cette version «traditionnelle » du Bumba Boi soit un produit adapté pour distraire - et même glorifier- l'élite blanche brésilienne. Le groupe de vachers et surtout leur maître, sont toujours les personnages les plus richement vêtus de strass, tandis qu'on leur donne de toute évidence le rôle de gardiens de l'ordre économique et moral, face au couple de paysans noirs qui est, quant à lui, décrits comme paresseux, maladroits et voleurs, et à qui l'on attribue le rôle des bouffons de la comédie. Enfin, le rôle des Indiens peu vêtus et enrubannés de plumes, est souvent tenu par des jeunes et jolies danseuses métisses à qui, pour l'occasion, l'on attache quelquefois de fausses tresses dans leurs cheveux crépus.

Le couple de bouffons n'est cependant pas considéré comme esclave, mais il correspond plus étroitement à une caricature des petits paysans libres de l'époque coloniale (esclaves affranchis ou indigènes métis). Les « travailleurs libres " du XIX ${ }^{e}$ siècle étaient accusés par l'élite blanche d'être paresseux, incapables d'initiative, se contentant de vivre dans la misère mais à proximité des grandes plantations ou des fazendas d'élevage, puisque leur principal moyen de subsistance aurait consisté à voler dans les champs des autres, voir 
aussi, à voler du bétail! Regina Prado a retrouvé une chronique journalistique, datant de 1855, qui employait précisément les termes de "paresseux voleurs " à propos de ces «travailleurs libres » de l'époque, c'est-à-dire l'ensemble de la population non esclave et non blanche du XIX ${ }^{e}$ siècle, composée d'indigènes pacifiés, de métis et d'anciens esclaves Noirs affranchis, c'est-à-dire finalement l'ensemble des ascendants de la société paysanne actuelle (Prado $1977: 167$ ). Ces stéréotypes furent tellement tenaces que, selon Alfredo Wagner Almeida, les livres d'histoire régionale du Maranhão ont accusé ces petits paysans métis d'avoir été responsable du retard économique de toute la province et de la crise que provoqua un manque récurrent de "main d'œuvre » disponible (Wagner 1983). Ainsi, la comédie «traditionnelle » du Bumba Boi a cristallisé ces préjugés raciaux issus de cette époque, à partir de l'angle de vue de l'élite blanche qui cherchait (et cherche encore largement aujourd'hui) à justifier un clivage social à l'aide d'un marquage racial et stéréotypé des comportements de chacun.

Si cette comédie "traditionnelle » était restée le mobile central des fêtes de Bumba Boi, on comprendrait mal comment une telle manifestation consistant finalement à glorifier l'élite blanche au détriment de sa population paysanne « libre » et métisse, soit devenue la manifestation " populaire " la plus intense et la mieux suivie à São Luís tout comme dans les zones rurales du Maranhão. Tout au plus l'aspect largement raciste et désuet de la comédie peutil expliquer la raréfaction des représentations de sa version « traditionnelle » au bénéfice aujourd'hui d'un développement plus important de sa dimension musicale et simplement festive. De ce fait, dans les grandes villes comme São Luís, où l'on fait appel à des troupes spécialisées afin d'animer les places publiques et les lieux touristiques, cette dimension musicale est explicitement devenue un critère fondamental d'appréciation. Les troupes sont communément classées en trois styles, selon leur caractéristique instrumentale : En premier lieu, le style "Boi zabumba », du nom du tambour bi-membrane, est considéré le plus traditionnel bien qu'il soit très certainement aujourd'hui le moins prisé des trois. Il est vrai que les battements lents des tambours zabumbas donnent à la manifestation un caractère monotone, auquel le public ne participe généralement pas ou peu. Le style "Boi matraca " est incontestablement le style le plus apprécié, pour une raison simple: n'importe qui peut acquérir deux morceaux de bois (matracas) et se mêler à la troupe des musiciens afin de participer à la frénésie collective. Dans les quartiers pauvres de la ville de São Luís, l'immense majorité des hommes possèdent effectivement une paire de matracas à demeure, réalisée à partir d'un bois dur et poli. Enfin, le style "Boi orchestra » est, comme son nom l'indique, un genre de scène qui a démultiplié la diversité instrumentale en ajoutant cuivres et cordes et, surtout, en s'agrémentant d'une amplification électro-acoustique. Ce style, qui est plus lyrique, est particulièrement bien adapté pour les spectacles touristiques et les animations des grandes places publiques, du fait qu'il utilise invariablement l'amplification stéréophonique, ce qui donne aux chanteurs un rôle privilégié et au public un stricte rôle de spectateur.

Parallèlement à cette évolution du Bumba Boi vers des formes musicales plus aptes à attirer les foules urbaines, dans les zones rurales par contre c'est la comédie qui a surtout évolué vers des formes plus aptes à 
s'accorder à une réalité locale contemporaine. En effet, au sein des villages, quand les paysans jouent cette comédie pour eux-mêmes, l'action burlesque, au lieu de disparaître comme en ville au profit de la musique, s'est considérablement développée en abandonnant l'essentiel de la version " traditionnelle", hormis les costumes et, bien entendu, le masque du bœuf. Ce développement, qui s'apparente plutôt à une rupture, consiste non seulement à inverser ra satire comique mais surtout à se réapproprier le thème de la comédie afin d'accorder une large place à l'improvisation et à la création locale.

Hormis les costumes et le masque du bœuf, les représentations rurales ont gardé de la comédie " traditionnelle » leur structure dramatique, mais leur genre comique s'articule maintenant sur le mode de la caricature de certains traits ou de faits réels, contemporains et éprouvés par les groupes. De cette manière, lors de chaque représentation dans leur contexte rural et domestique, les nouvelles comédies de Bumba Boi prennent l'allure d'une revue des évènements vécus par chaque groupe durant l'année écoulée. Libérés de leur contenu " traditionnel », les personnages des comédies rurales excellent dans l'art de la satire qui, basée à l'origine sur des stéréotypes raciaux, visent maintenant à dénoncer les conflits sociaux les plus immédiats. Sur base d'un fond musical commun, chaque petite troupe se crée son propre répertoire de chants et chaque année de nouveaux thèmes satiriques sont choisis et à moitié improvisés. Même si les événements qui inspirent chaque nouvelle création changent d'année en année et que, de ce fait, l'intrigue est à chaque fois différente, le bœuf réapparaît invariablement comme l'élément central des récits et continue à jouer le rôle d'objet convoité et de litige entre les personnages. Aussi, même si le vol du bœuf est resté une constante dans les comédies, ce n'est plus à la catégorie raciale «nègre » que l'on attribue le méfait, ni non plus à la catégorie sociale du "riche maitre blanc " que l'on accorde le plus bel apparat en même temps que le statut de gardien de l'ordre moral. Au contraire, en s'appropriant l'action dramatique, le vol du bœuf est devenu l'élément déclencheur d'une critique sociale qui, sur le ton de la farce, expose les tensions quotidiennes au cour parfois des conflits sociaux les plus intenses.

Mundinha Araujo, dans un relevé ethnographique réalisé à la veille d'une large expropriation collective dans le nord de la région d'Alcântara (pour y installer une base spatiale et militaire), releva cette année-là dans un des villages concernés le texte de leur comédie du Bumba Boi (Araujo 1990). La scène mettait face à face un homme inconnu (mais représentant vraisemblablement l'Autorité publique), qui vint se présenter aux paysans pour leur annoncer qu'il désirait s'approprier leur lieu. Le texte caricaturait judicieusement le sentiment d'incompréhension générale vécu par les paysans cette année-là qui, bien qu'informés de leur imminente évacuation par les Autorités, étaient vraisemblablement désemparés et bien indisposés à comprendre le mobil de cette expropriation. Dans leur comédie, cette situation vécue comme absurde était judicieusement rendue en caricaturant la "demande" de l'homme inconnu: la comédie débutait au moment où ce personnage se présenta dans le village en fête et interrompit la musique en affirmant qu'il désirait «acheter, louer, emprunter ou recevoir... le périmètre de la danse (o terreiro) ». Incapable d'être satisfait par une demande aussi absurde, 
l'inconnu leur vola finalement le bœuf. Dans cette version de la comédie du Bumba Boi, c'est très clairement l'expropriateur qui prenait le rôle de bouffon ridicule face aux agriculteurs désemparés qui, eux, portaient les vêtements des vachers de la comédie «traditionnelle » ${ }^{3}$.

Regina Prado, dans 1'unique étude anthropologique consacrée à cette fête en milieu rural, a relevé une version où c'est " un prêtre missionnaire étranger " qui tenait le rôle du bouffon à qui l'on attribua également le vol d'un bouf (Prado 1977). Cet élément original s'explique par le fait que le village dans lequel se joua cette comédie était cette année-là en train de vivre un conflit avec la paroisse de São Luís, légitime propriétaire des terres occupées par le village. La terre appartenait sans doute en droit à l'Eglise depuis plusieurs siècles, mais en l'absence de toute expression tangible de ce droit, les habitants considéraient eux depuis déjà plusieurs générations que le légitime propriétaire de leur terre était leur Sainte patronne à qui ils " payaient » une fête chaque année...

Enfin, dans une version mieux connue dans toute la région, l'action burlesque oppose un groupe de paysans désemparés face aux exigences administratives de la sécurité sociale brésilienne lors de l'obtention d'une allocation de pension de survie ou d'une aide pour des frais d'obsèques. Pour saisir ici la caricature, il faut avoir connaissance du fait que la loi brésilienne prévoit normalement d'accorder à tous les travailleurs ruraux âgés de plus 60 ans et sans ressources, ex-salariés ou non, une pension de survie équivalant à un salaire minimum (en 1996, environ 90 Euros mensuels). L'Etat prétend également prendre à sa charge les frais d'obsèques de ces pensionnés décédés (et donc régulièrement inscrits comme tels). Dans cette comédie satirique du Bumba Boi, l'action burlesque débute par la mort inopinée d'un vieillard précisément au moment où celui-ci entama la procédure de demande d'une pension de survie : le vieil homme décéda au moment où l'on tira sa photo... De ce fait, il n'était plus question de recevoir une pension de survie et le groupe qui accompagnait le défunt demanda plutôt à cette même Administration une allocation de frais d'obsèques. Mais celle-ci leur fut aussitôt refusée puisque, pour l'Administration, le défunt n'était pas encore régulièrement inscrit comme un pensionné nécessiteux (qui est la condition légale pour que l'Etat accorde de prendre à sa charge les frais d'obsèques)... Du coup, pour parvenir à payer les funérailles, le petit groupe de paysans vola le bœuf de la fazenda (celle-ci n'est pas identifiée par les comédiens). Dans cette comédie, fort drôle, non seulement le conflit n'a pas d'issue (le bœuf est vendu pour payer les obsèques), mais on doit surtout constater que "la fazenda", bien privé, est assimilée à l'Administration des pensions, à l'Etat, c'est-à-dire au pouvoir politique et financier en général (et sans doute implicitement blanc).

Regina Prado a analysé la structure générale de ces comédies rurales en relevant l'opposition relationnelle qui semble systématiquement animer un groupe de dirigeants (les mandates, c'est-à-dire le groupe des vachers, représentant de l'ordre et de la norme) et celui du bouffon (palhaço). L'auteur relève que même si le rôle du bouffon consiste systématiquement à se comporter et à dire tout le contraire du normal, de l'attendu ou du moral, celuici est malgré tout utilisé comme un véhicule d'expression des vérités telles qu'elles sont perçues par les paysans (Prado 1977:177-178). Aussi, selon Prado, ce 
paradoxe entre forme mensongère et contenu véridique, s'expliquerait par le fait que la normalité et la folie sont toujours définies par un code dominant, c'est-à-dire "par un code qui pour être celui de la classe sociale qui est ellemême dominante, agit également comme élément de domination des autres classes sociales » (Idem, ma traduction). Or, selon l'auteur, grâce au fait que la critique exposée par le bouffon soit justement formulée par un être transgresseur, cela permet de donner à cette critique une existence à l'intérieur d'un cadre de vérité qui, autrement, lui donnerait d'emblée le statut de mensonge. En d'autres termes, c'est parce que le bouffon est un menteur que ses propos "mensongers" peuvent être légitimement formulés. Son rôle consisterait finalement "à dire des vérités sur le ton du mensonge " (Prado 1977:182)

Cette analyse, quoique fort séduisante, repose abusivement sur l'idée que l'opposition entre le groupe des vachers et celui du bouffon reproduit implicitement une opposition de classe entre l'élite blanche, héritière du pouvoir colonial, et les paysans noirs, héritiers de la servitude. Il est indéniable que la comédie du Boi sert souvent d'instrument de dénonciation politique; mais il n'est pas moins fréquent que cette dénonciation passe par la voix du chanteur principal qui, dans la comédie, tient systématiquement le rôle de chef des vachers (et de la danse) et non pas celui du bouffon. Regina Prado rapporta elle-même l'exemple d'un de ces chanteur de Bumba Boi qui aurait subi une persécution physique allant jusqu'à un emprisonnement, parce qu'il avait exprimé trop ouvertement l'un ou l'autre méfait perpétré par un puissant homme politique local (Prado 1977:200). Dans le contexte pré-démocratique de l'époque à laquelle la thèse de Regina Prado fut écrite, on peut comprendre le besoin de camoufler un discours politique derrière une satire comique aux allures bouffonne et «bon enfant». Dans ce contexte effectivement, sans doute que l'analyse de l'auteur devait être partiellement exacte. De ce fait, il est probable que la ré-appropriation de la comédie «traditionnelle » du Bumba Boi au profit d'une satire sociale réactualisée, ait été amorcée dans ce contexte de la dictature militaire, au même moment où la jeunesse intellectuelle et urbaine du pays utilisait quant à elle la musique pop comme vecteur d'expression de son opposition au régime. Mais depuis, le contexte a changé, la liberté d'expression s'est étendue à toutes les couches sociales au point qu'il est devenu aujourd'hui courant (et même banal) d'entendre ouvertement traiter les hommes politiques de "voleurs». Ce changement survenu dans l'espace social brésilien n'a cependant pas affecté le Bumba Boi au point de lui faire perdre son style comique et dénonciateur. Par contre, ce qui a sans doute évolué depuis, c'est l'amplitude de la rupture entre la comédie "traditionnelle " et les comédies actuelles. Aujourd'hui, les vachers et leur chef peuvent représenter les intérêts des paysans et le bouffon peut tenir le rôle d'un homme puissant. Dans ce cas, la critique devient carrément explicite puisque ce sont les paysans qui sont présentés comme sérieux et "normaux" tandis que le bouffon tient le rôle d'une autorité ridicule et insensée.

La comédie du Bumba Boi a donc considérablement renforcé son rôle de contrôle social des évènements vécus par la population paysanne et appréhendés à sa manière. Aussi, ces représentations théâtrales s'articulent 
toujours à partir d'une dimension relationnelle qui oppose le mode de penser et d'agir des uns face au mode de penser et d'agir des autres. Or, il est significatif à cet égard de relever le fait que ces comédies ne traitent jamais de problèmes strictement domestiques ou même villageois, mais préfèrent au contraire mettre en scène des relations conflictuelles entre un groupe domestique et un élément extérieur à celui-ci (le propriétaire terrien, l'Autorité publique, le missionnaire,...). En ce sens, la comédie du Bumba Boi peut être considérée comme le lieu de production et d'expression d'une éthique commune ou encore, comme le théâtre d'une identité paysanne locale mesurée face à une altérité globale et diffuse. Cette "identité " s'oppose cependant à l'interprétation classique d'une "identité traditionnelle » ou «ethnique » pour se présenter davantage ici comme un discours dynamique -et problématiquesur le quotidien; un discours revu chaque année et sans cesse renouvelé en réaction aux événements qui sont ressentis comme des tensions objectives du groupe et qui, de ce fait, permettent d'opposer un " nous » à un « autre ».

Cette révolution de la comédie du Bumba Boi produit de ce fait un paradoxe qui ne peut complètement échapper aux acteurs : alors que d'un côté cette tradition se présente de plus en plus clairement comme un mécanisme d'émancipation d'une éthique paysanne, de l'autre, les artifices qu'elle emploie pour y parvenir s'appuient sur une appropriation des personnages représentant à l'origine une allégorie de l'élite coloniale, esclavagiste et dominatrice. Mais dans les comédies contemporaines, non seulement le groupe des Indiens tend à disparaître, mais aussi celui des "paysans libres " originellement représentés par la figure du bouffon à tête de Nègre. En outre, si le rôle du bouffon est toujours bien présent dans les comédies actuelles, celui-ci est de plus en plus détaché de tout stéréotype racial ou même social prédéfini. Seule persiste la figure du guignol qui peut, selon les comédies et d'une année à l'autre, se présenter sous les traits d'un prêtre, d'un fonctionnaire public, d'un riche fazendeiro, d'un commerçant ou d'un simple inconnu, tout comme sous les traits d'un paysan local et proche (cf. supra, l'exemple du vieillard décédé). En réalité, l'opposition relationnelle entre le bouffon et les vachers, bien que toujours fondamentale dans l'articulation des intrigues, $n$ 'est plus figée sur une critique sociale de type raciale ou ségrégationniste. Les personnages des vachers jouent souvent aujourd'hui -mais pas systématiquement non plus- le rôle des paysans locaux (cf. supra, exemple de l'expropriation collective), tout comme ils peuvent reprendre et incarner dans d'autres comédies le rôle d'une autorité extérieure (Etat, propriétaire terrien). Cette décaractérisation progressive du mythe colonial originel (les trois races réunies sous l'autorité du maître blanc et catholique) implique que beaucoup de petites troupes rurales n'ont gardé comme seul costume spécifique que celui des vachers enrubannés. La référence implicite à l'époque coloniale n'existe donc plus aujourd'hui que dans la tête des anthropologues, des historiens ou des folkloristes. La fonction dramatique des vachers doit finalement être mesurée non plus aujourd'hui en fonction de ce que ces personnages représentaient dans le mythe historique, mais par ce qu'ils font concrètement dans toutes les comédies : ils font danser un bœuf. 


\section{LE BCEUF ET LA FIGURE SAINTE}

Concevoir la tradition du Bumba Boi comme s'il s'agissait d'une fresque historique rejouée chaque année, risque de nous éloigner du mobil existentiel et collectif qui anime cette fête. De même, focaliser notre attention sur la comédie et plus particulièrement sur la satire politique qu'elle abrite souvent, peut également nous tromper. Ces deux approches considèrent la figure du bœuf comme étant relativement secondaire, ce qui contredit pourtant le message explicite de cette manifestation. En effet, quel que soit l'engouement, la frénésie, l'ivresse ou le burlesque des personnages et de leur public, cette fête consiste d'abord à se dépenser pour faire danser... un bœuf. Or, même si elle suscite la passion, cette dépense souvent très importante (à coup de riches costumes, de plantureux repas et surtout de beaucoup de boissons), se justifie d'abord et surtout -de l'avis de tous- par le fait que la danse du bœuf est réalisée par obligation religieuse, pour Saint Jean, considéré comme le saint patron de cette fête.

L'attention de la plupart des folkloristes a été détournée de cette dimension religieuse essentielle, certainement en raison du fait que la fête du Bumba Boi est réalisée dans d'autres régions du Brésil, sous des formes légèrement différentes où elle est liée à d'autres fêtes calendaires, comme la fête des Rois (Carvalho 1995). L'association entre le Bumba Boi et Saint Jean Baptiste (São João Baptista) en particulier, ne semble donc relever d'aucune nécessité, d'autant que d'autres saints catholiques, tels que São Pedro et São Marçal, sont quelquefois également invoqués dans le Bumba Boi et peuvent même se substituer à St Pierre. En outre, les hagiographies tant officielle que populaire ne semblent pas évoquer de lien significatif entre cet animal particulier et l'une de ces figures saintes. Il importe donc de saisir la nuance: Saint Jean n'est pas considéré comme étant associé aux bœufs mais comme adorant particulièrement le Bumba Boi, ni l'animal, ni ses comédies, mais tout simplement sa fête.

Les fêtes du Bumba Boi sont insérées dans un calendrier cérémoniel, qui l'on nomme officiellement «les fêtes de juin » (festejos juninos), bien qu'elle ne finissent que vers septembre ou même, au plus tard, à la Toussaint $\left(1^{\text {er }}\right.$ novembre). Ces «fêtes de juin » sont explicitement liées à la figure de Saint Jean (fêté le 24 juin), lié au changement de saison (solstice d'été) et sans doute aussi à l'idiome du feu : ces manifestations sont régulièrement qualifiées de "flambée " (fogueira), et s'inscrivent au début d'une saison de brûlis (on parlera des "essarts de la St Jean»). Le feu est également un élément matériel incontournable à toutes ces fêtes qui utilisent le tambour ou le tambourin comme instrument principal, car leurs membranes de cuir sont toujours relativement peu tendues sur leur socle de bois et il s'avère nécessaire de régulièrement les réchauffer -en les approchant d'un feu- afin de retendre les peaux et d'obtenir le claquement sonore désiré. De ce fait, les nuits de fête comme celle de la Saint Jean, de multiples feux jalonnent effectivement toutes les villes et les villages de la région.

Les «fêtes de juin » débutent en réalité un mois plus tôt, en mai, par une période de répétitions ou d'essais (ensaios), durant laquelle les troupes de 
Bumba Boi ne s'éloignent généralement pas de leur environnement villageois (ou, en ville, de leur quartier). Cette période de préparation consiste non seulement à composer de nouveaux chants et à choisir de nouveaux thèmes pour les comédies, mais surtout à réunir les troupes, à rassembler les moyens nécessaires pour confectionner ou réparer les costumes et les instruments et, enfin, à préparer le masque du bœuf. Parmi les petites troupes des zones rurales du Maranhão, cette période d'ensaios consiste surtout à concrétiser la formation d'un groupe de danseurs qui, en y participant, s'engageront à conduire le Boi devant la figure de Saint Jean, la nuit du 23 au 24 juin. Beaucoup de troupes de Bumba Boi sont généralement formées autours d'un noyau de quelques individus -toujours des hommes- qui, d'année en année, tentent de conduire le groupe entier, composé également de nouvelles recrues qui, par amitié, par plaisir ou par dévotion à Saint Jean, s'engagent à y participer au moins le temps d'une saison.

L'histoire de chaque troupe débute invariablement par la réalisation $\mathrm{d}^{\prime}$ une promesse faite par un seul individu à Saint Jean en échange d'un vœu qui se serait effectivement réalisé (le plus souvent une guérison, mais aussi une réussite en affaire). Faire une " promesse » à un saint particulier et, par la suite, lui « payer » cette promesse (pagar a promessa) sous forme d'une fête réalisée en son honneur une fois le vœu exaucé, constitue le mode par lequel les paysans conçoivent le culte des Saints catholiques. Cela ne se limite pas au Bumba Boi ni à Saint Jean. En dehors du Carnaval (qui est une fête de grandes villes), toutes les fêtes locales sont dédiées à une figure sainte et la plupart ont effectivement une promesse pour origine. Aussi, dans cette société, la fête s'apparente à un moyen d'échange entre les hommes et les saints catholiques, servant concrètement à " payer les promesses » faites aux saints et donc à rétribuer leurs actions divines, leurs miracles.

Le fondateur d'une troupe de Bumba Boi est donc un promesseiro (un "prometteur "), c'est-à-dire quelqu'un qui a réalisé une promesse et qui, pour payer sa dette à Saint Jean, doit former une troupe de Bumba Boi qu'il conduira devant la figure sainte la nuit du 23 au 24 juin. Pour ce fondateur, un tel engagement consiste à réunir des partenaires (danseurs et musiciens) qui accepteront de le suivre soit par égale dévotion à Saint Jean, soit pour simplement bénéficier des plaisirs de la fête, soit encore par solidarité vis-à-vis de lui (un parent ou un compère) mais plus généralement encore, pour ces trois raisons cumulées. Durant la période des essais (ensaios), les troupes se réunissent généralement une soirée par semaine durant laquelle le dirigeant de la troupe (son fondateur ou son remplaçant) doit offrir des boissons et un repas à tous les participants (musiciens et danseurs). Bien que ces "essais " se déroulent habituellement dans leur contexte domestique (lieu de résidence de son «prometteur"), ils sont toujours suivis par le voisinage, heureux de venir s'y amuser. De ce fait, certaines troupes parviennent déjà à produire quelques bénéfices durant cette période en s'associant à un vendeur de boissons qui partagera ses recettes avec la troupe. En règle générale cependant, dans les zones rurales les dépenses de la troupe elle-même sont toujours plus importantes que les recettes de sorte que, pour le dirigeant comme pour les danseurs costumés, la participation à une troupe est invariablement considérée 
comme une dilapidation de son épargne que seule une obligation religieuse peut suffire à justifier. La formation d'une troupe repose donc, chaque année, sur la capacité et la volonté d'un nombre restreint de personnes (environ une douzaine au minimum) prêtes à dépenser ensemble des moyens importants dans la confection et l'entretien des costumes, la construction et la décoration d'un nouveau masque et, surtout, l'organisation de banquets (en début et fin de cycle). Pour cette raison, en dehors de quelques notables exceptions, beaucoup de petites troupes de Bumba Boi apparaissent et disparaissent chaque année, au gré des promesses et des capacités d'épargnes des dévots à Saint Jean. Dans la plupart des villages, une troupe peut se former chaque année, tout comme il peut se passer de nombreuses années avant qu'il s'en reforme une nouvelle.

Bien que ce ne soit pas leur fonction originelle, certaines troupes de Bumba Boi réapparaissent systématiquement à chaque saison et parviennent donc à devenir permanentes. Dans ce cas, l'acte de fondation initial, toujours basé sur une promesse individuelle précise, se meut en une véritable vocation composée à la fois de dévotion religieuse, de passion pour le Boi (ses danses et/ou ses comédies) et de professionnalisme. Dans ce cas également, la troupe se dote d'une meilleure organisation et la charge du fondateur peut se transmettre à un proche (déjà membre de la troupe) ou même être héritée après sa mort. Ces troupes professionnelles restent généralement liées à l'unité résidentielle de son fondateur (un village ou un quartier), mais bénéficient d'une réputation qui s'étend quelquefois à tout l'Etat. Aussi, après la période des "essais ", c'est-à-dire juste après la nuit de la Saint Jean, ces troupes réputées parcourent toute la région (et même le pays) pour réaliser des spectacles de comédies (troupes rurales) ou uniquement de musiques et de danses (troupes urbaines). Ces véritables tournées peuvent durer plusieurs mois avant de libérer leurs membres qui, de ce fait, perçoivent quelquefois un salaire ou se partagent les bénéfices de chaque représentation.

Quel que soit leur niveau de permanence et de professionnalisme, les troupes de Bumba Boi ne semblent jamais complètement basculer dans une logique strictement économique guidée par le seul profit de ses membres. L'orientation religieuse reste effectivement très présente et la plupart des troupes permanentes ne se considèrent pas comme de véritables compagnies mais plutôt explicitement comme des "confréries" (irmandades) ${ }^{4}$. Dans la région, ce terme de "confrérie " s'applique effectivement à toutes les associations qui se donnent pour charge l'organisation d'une fête de saint patron. Dans ce contexte, les troupes de Bumba Boi sont donc considérées comme de simples confréries spécialisées dans le culte de Saint Jean, qui ont toutefois la particularité d'être normalement momentanées (puisque liées à des promesses individuelles), contrairement aux confréries classiques qui se fondent d'emblée sur une permanence de leur culte. C'est parce que les troupes de Bumba Boi sont assimilées à des confréries que de nombreuses personnes apportent spontanément leur soutien matériel à ces associations festives et pieuses, sans pour autant y participer activement ni entretenir de relation personnelle avec ses membres, outre la co-résidence de village ou de quartier.

Normalement, même si la troupe parvient à se pérenniser, chaque année le masque du bœuf doit être baptisé d'un nouveau nom et recouvert d'un 
nouveau «cuir» (couro): l'ossature en bois léger, munie d'une tête et parée de vraies cornes, est enrobée d'un tissu neuf en feutre noir et décorée de milliers de petites perles multicolores formant un motif quelconque et composant souvent son nouveau nom. Comme les troupes pérennes ne sont plus directement et personnellement engagées par une promesse à Saint Jean, certaines d'entre elles s'associent à un couple de "prometteurs » (promisseiros) qui, pour avoir euxmêmes réalisé une promesse à Saint Jean, prennent alors une partie de la charge normalement dévolue à son fondateur (nouveau cuir, repas lors des ensaios, etc). Ces individus qui ne font donc pas directement partie de la troupe des danseurs et musiciens professionnels, deviennent alors les padrinhos do Boi (le parrain et la marraine du Bœuf). Dans tous les cas, les troupes organisent un baptême, inspiré d'une cérémonie classique, consistant à oindre le masque en lui donnant pour la première fois son nouveau nom et en dévoilant au public son nouveau «cuir» qui durant toute la période des essais était dissimulé sous un linge ${ }^{5}$. La cérémonie du baptême, accompagnée d'un repas offert par le couple de parrains, s'exécute à l'aide de nombreuses prières catholiques (Ave-Maria, PaiNosso) dont évidemment des oraisons à Saint Jean-Baptiste. D'autres dévots ont coutume de participer à cette cérémonie tout comme ils participent aux autres fêtes de saints organisées par leur entourage, en offrant une contribution aux frais du banquet ou en allumant simplement un cierge.

La cérémonie du baptême, réalisée la veille de la Saint Jean, clôt la période des ensaios et, avec elle, les manifestations domestiques du Bumba Boi (dans leurs villages ou leurs quartiers respectifs). Durant la même nuit, ou plutôt à l'aube du 24 juin, toutes les troupes se dirigent en procession vers l'effigie du saint afin d'accomplir toutes les promesses qui se sont associées pour former les troupes. Quelques confréries importantes implantées dans leur village possèdent leur propre statuette du saint, logée dans une chapelle spécialement érigée par eux. Cependant, depuis quelques années semble-t-il, la grande majorité des troupes de Bumba Boi choisissent d'organiser leur procession vers une effigie logée dans une église publique où se rendent la plupart des autres Boi de leur région. De nombreux officiels de l'Eglise catholique n'apprécient pas cette manifestation "populaire». A São Luís par exemple, l'accès à l'Eglise de Saint Jean, située en plein cour de la capitale, leur a été interdite. Mais curieusement, l'accès à la chapelle de Saint Pierre, située à l'entrée de la ville, leur a été récemment concédé ${ }^{6}$. Cette substitution forcée ne semble pas poser de problème aux danseurs du Bumba Boi dans la mesure sans doute où Saint Pierre fait également partie du cycle des fêtes de juin. Surtout, l'effigie de Saint Pierre qui se situe dans la chapelle de São Luís, fait partie des statuettes les plus adorées de la région car cette figure catholique est également considérée comme le patron des pêcheurs, nombreux à São Luís. Ainsi, chaque année, tout au long de la nuit du 23 au 24 juin, des centaines de troupes de Bumba Boi assiègent la capitale du Maranhão, certaines ayant parcouru plusieurs dizaines de kilomètres à pied durant toute la soirée, afin de se présenter devant cette idole catholique logée dans une chapelle située à l'entrée du vieux port. Durant cette fastueuse nuit, sans doute à la faveur de la frénésie collective, de la fatigue et de l'alcool, la plupart des acteurs se laissent emporter par leur jeu: lorsque chaque masque du bœuf entre dans la chapelle 
accompagné de sa troupe, et que son museau vient baiser les pieds de l'idole tout en dansant devant elle, des dizaines de personnes qui accompagnaient la troupe se précipitent alors pour caresser l'animal, le toucher, comme si l'objet qui le représente était tout à coup investi d'une puissance bienfaisante.

Bien que cette procession devant la figure sainte constitue formellement le début de la période des festivités publiques (tournées de spectacles de danse et/ou de comédie en dehors des villages ou des quartiers), elle se présente également comme le moment où la performance atteint son maximum d'intensité (son climax), en raison sans doute du fait qu'il s'agit du moment solennel où s'accomplissent les promesses qui sont à l'origine de chaque troupe. Aussi, s'avancer devant l'effigie s'apparente à un aboutissement, l'objectif de chaque promesse, qui renferme la somme des efforts et des sacrifices matériels mis en ceuvre pour faire danser le masque. On peut sans doute comprendre que le geste qui consiste à toucher le masque au moment précis où il danse sa révérence au pied de la figure sainte, soit effectivement vécu comme magique dans la mesure où il matérialise le contact recherché avec la puissance sainte. Situé dans le prolongement de la promesse payée, ce geste indique également l'importance accordée au masque lui-même et surtout à sa fonction de médiation entre le "prometteur » et la puissance divine: ce n'est pas la statuette sainte qui est touchée par la foule, mais bien le masque, c'est-à-dire le médium de cette relation. En outre, tout se passe comme si, dès cet instant, un mouvement inverse s'amorçait : jusqu'ici les hommes s'étaient organisés pour conduire leur masque, en dansant tous en direction de la même effigie sainte. Jusque-là on pouvait sans doute penser que c'était cette figure catholique qui orientait toutes les troupes, tandis que depuis ce moment c'est indubitablement chaque masque médium qui oriente, à lui seul, toute l'attention des troupes et de leur public. Chaque masque du bœuf, à la fois délivré de ses promesses et investi d'une puissance que l'on cherche à s'approprier par contact, sort de la chapelle suivit par tous les fidèles de sa confrérie pour une nouvelle période de fête, de danses et de comédies publiques.

La période des tournées publiques peut être plus ou moins longue et intense selon la renommée de la troupe et les mouvements de foule qu'elle est capable d'attirer à elle. Il est difficile d'apprécier dans quelle mesure la performance musicale et chorégraphique ou, plutôt, le fond lyrique, satirique ou même politique des textes, peuvent expliquer le plus ou moins grand succès de certaines troupes. Il est évident qu'un bon ensemble se doit d'allier tous ces aspects à la fois. Même parmi les troupes qui favorisent les spectacles de musique et de danse au détriment de la comédie, l'élément satirique des intrigues du bœuf réapparaît alors souvent dans les textes de leurs chansons. Aussi, même dépouillé de toute forme théâtrale, le Bumba Boi maintient généralement dans ses chants une dimension "éthique » ciblée sur une critique sociale amusante ou un jugement moral. De même, les troupes qui favorisent la comédie, organisent des spectacles qui durent de longues heures car, outre leur déroulement dramatique et satirique, ils accordent également toujours de longs moments aux musiques et aux danses auxquelles participe toujours l'ensemble du public. 
Les performances musicales du Bumba Boi alternent donc deux dimensions qui sont ici inconciliables : la musique que l'on danse et la musique que l'on écoute, soit respectivement, le rythme et les mots. Quand tous les instruments de percussion battent ensemble, il n'est plus possible d'entendre le chanteur. Dès lors, ce dernier ne chante que quand les instruments se taisent et que tout le monde l'écoute. Aussi, la performance musicale-qu'elle agrémente une comédie ou qu'elle constitue le but de la manifestation, est toujours structurée par une alternance entre la voix du chanteur et les battements des percussions, à laquelle correspond une alternance entre l'écoute immobile et la danse du public. C'est le chanteur principal (qui dans la comédie traditionnelle tient le rôle de l'intendant de la fazenda et, dans tous les cas, le garant du «bon sens " local) qui invariablement mène le jeu de cette alternance : les musiciens attendent qu'il ait terminé sa strophe et qu'il entame le refrain pour reprendre leurs percussions jusqu'à couvrir son chant et entraîner le public avec eux. De même, ce chanteur arrête les instrumentistes (et le public) dès qu'il désire reprendre son texte (d'un geste de la main ou à l'aide d'un sifflet). Quelquefois, on a l'impression que le chant n'est là que pour permettre aux batteurs de se reposer et suriout de leur donner le temps d'aller vite retendre les membranes de leurs tambours devant un feu. Dans ce cas, c'est bien le rythme et la danse qui dominent le Bumba Boi. Mais à d'autres moments, le chanteur prend largement le dessus de la troupe en déclamant une longue logorrhée, souvent drôle, tantôt poétique, tantôt politique, parfois en vers, parfois à peine mélodique, en ne laissant que peu de place aux tambours et à la danse. Dans ce cas, c'est la parole et l'écoute immobile qui dominent la réjouissance publique. Le succès d'un spectacle de Bumba Boi repose finalement sur ce jeu de performance et de contre-performance entre le chanteur et ses musiciens, entre la sémantique de la parole et le mouvement rythmique des corps. Or, ce jeu de performance et de contre-performance structure en quelque sorte ici son espace politique: l'entrain de la danse permet d'unir une foule désorganisée qui, convertie en assemblée, est invitée entendre le message du chanteur ou du comédien.

Enfin, le cycle complet des festivités du Bumba Boi s'achève par un retour des troupes dans leur espace domestique et la réalisation d'une ultime fête qui se clôture par la mise à mort rituelle de l'animal ( $a$ matança). Cette cérémonie, qui prend clairement des allures d'un sacrifice, peut se dérouler à n'importe quel moment après la nuit de la Saint Jean, dès que la troupe décide de cesser ses tournées, au plus tard le $1^{\mathrm{er}}$ novembre dit-on, le Jour des morts.

On pourrait aisément gloser sur cette mort symbolique en y voyant, par écho à la cérémonie du baptême qui initie la première sortie publique, le fondement structural de toute la manifestation. On pourrait aussi y voir un fondement christique quand, au moment de trancher la gorge de la victime, le "sang " de l'animal est récupéré dans une vasque (on fait couler du vin) puis distribué en boisson à tous les membres de la troupe... Mais, comme l'avait déjà noté Regina Prado, il convient sans doute ici d'attacher au moins autant d'importance au plaisir de l'acte "profane " consistant à simplement boire un bn coup de vin (Prado 1977:189-192). Il n'y a effectivement pas de multiples façons de clôturer un cycle de fête qui s'est justement choisi comme effigie un animal d'élevage. 
Les références explicites au modèle chrétien, tout comme la recherche d'une cohérence rituelle et d'une certaine solennité, ne sont peut-être ici que des vecteurs d'une légitimité, d'une recherche d'un sentiment de vrai et $\mathrm{d}^{\prime}$ authentique, sans pour autant devoir devenir le fondement de toute la manifestation. Aussi, la mise à mort de l'animal, destin normal de tout animal d'élevage, expose surtout la nécessité d'aboutir, de rendre à chacun son destin, y compris celui des membres de la troupe qui après de long mois de fêtes, pourront retourner à leurs occupations domestiques.

Il importe finalement de saisir dans l'aboutissement d'un cycle cérémoniel, les conditions de son renouvellement. La distribution du «sang " et quelquefois aussi le partage de la "viande " (l'ossature du masque) entre ses membres, renforcent sans doute le sentiment d'unité du groupe qui s'est constitué autour du masque et qui, de toute évidence, cherche à survivre audelà du sacrifice, c'est-à-dire au-delà du paiement des promesses individuelles et donc au-delà des motivations personnelles qui, ensemble, avaient produit un lien social. C'est donc aussi à ce moment précis de la fin du cycle cérémoniel que seront invitées à se déclarer les personnes qui prendront en charge le cycle festif de la prochaine saison. La mise à mort du Boi sert donc déjà à en faire naître un autre.

\section{CONCLUSION}

Le succès grandissant du Bumba Boi au Maranhão -qui est relevé par tous les observateurs locaux aujourd'hui- peut être mesuré tant au niveau de l'engouement des foules pour son divertissement (musiques, danses et comédies) qu'à celui de son engouement religieux (promesses à St Jean). Ce phénomène de passion collective doit être mis en relation de cause à effet avec une prise en charge de la manifestation par des unités sociales organisés -les " confréries »- qui tentent de produire et de reproduire d'année en année et de génération à génération, la pérennité de leurs liens sociaux. En d'autres termes, la population métisse du Maranhão ne serait parvenue à s'approprier la comédie et à réinventer son mythe, qu'à la condition d'être préalablement parvenue à éprouver le besoin de rendre pérenne ses formations sociales locales spécifiques.

En outre, l'identité de cette société métisse n'émerge pas à partir d'un accord immédiat sur les principes qui la fondent, mais au contraire dans le contexte d'une ré-appropriation du récit traditionnel de cette fête d'inspiration coloniale. C'est effectivement à l'aide d'une transfiguration des éléments officiels du mythe national brésilien -le Blanc, l'Indien et le Noir-que cette société métisse parvient à se penser comme une collectivité autonome au sein de l'espace national, et à ainsi se négocier un «nous ». Le Bumba Boi est donc le théâtre d'un découpage culturel sans cesse revisité par les acteurs d'une société qui se cherche dans le contexte d'une nation multiraciale. En paraphrasant JeanLoup Amselle (1990), on peut donc conclure que ce qui, dans cette "culture ", est transmis et devient donc pérenne, ce n'est ni la comédie des races, ni l'identité multiraciale ou multiculturelle du métis brésilien, mais les termes qui la fondent 
comme problème... Une identité peut donc être appréhendée collectivement sans

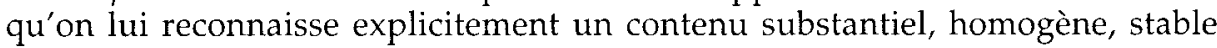
ou discret, à la condition que son groupe parvienne à se jouer lui-même. Dans ce contexte, nulle doute que le rite précède le mythe.

\section{BIBLIOGRAPHIE}

AMSELLE, J.L. 1990, Logiques métisses. Anthropologie de l'identité en Afrique et ailleurs. Payot, Paris.

ARAUjO, M. 1990, Breve memória das comunidades de Alcântara. SIOGE, São-Luís.

CARVAlHO, M. M. P. de, 1995, Matracas que desafiam o tempo: é o Bumba-Boi do Maranhão, um estudo de tradição / modernidade na cultura popular. São Luís. Thèse de Mestrado. UFRJ, Rio de Janeiro.

DAMATTA R. 1987, A Fábula das Três Raças ou o problema do racismo brasileiro. In DaMatta, Relativizando. Rocco, Rio de Janeiro.

PRADO R. de P. S. 1977, Todo ano tem. As festas na estrutura social camponesa. Museu Nacional, Rio de Janeiro.

WAGNER A. 1983, A ideologia da decadência: leitua antropologica a uma história da agricultura do Maranhão. IPES, São Luís.

\section{NOTES}

1. La version «traditionnelle", reprise par de nombreux folkloristes, est effectivement devenue "officielle " au Maranhão, depuis que le Théâtre de São Luís choisit de monter cette comédie populaire sous forme d'un opéra, comme première production originale après la restauration complète du bâtiment, en 1996.

2. Deux variantes de cette comédie urbaine, jouées chaque année par deux troupes des faubourgs de la capitale (São Luís) sont rapportées par Maria de Carvalho (1995). Dans l'une d'entre elles, ce n'est pas le docteur ou le chamane (pajé) qui sauve l'animal grièvement blessé, mais c'est Saint Jean qui offre un nouveau bœuf au fazendeiro en compensation de la perte de son bel animal.

3. Dans cette comédie, comme dans beaucoup d'autres de la région, le rôle des Indiens avait complètement disparu. Seul le rôle du pajé (chamane local mais non caractérisé d'Indien) s'est maintenu : il était fait appel à lui afin d'identifier le responsable du vol du bøuf (par divination), ce qui est aujourd'hui une fonction courante des guérisseurs pajés locaux.

4. Ce sentiment d'appartenir explicitement à une "confrérie " a été relevé tant par moi-même à Cururupú en 1995-1998, que par Regina Prado (1977) dans son étude d'une petite troupe rurale de 
la région d'Alcântara et par Maria de Carvalho (1995) pour deux grandes troupes urbaines de São Luís.

5.- Pour des raisons évidentes de reconnaissances de leur succès, les grandes troupes professionnelles qui sont réputées gardent le même nom chaque année, mais le cuir est par contre renouvelé. (Carvalho 1995:112-115). Inversement, faute de moyens, certaines petites troupes peuvent ne pas changer de cuir, mais bien de nom. En règle général, que ce soit l'un ou l'autre élément qui est renouvelé, tout le monde s'accorde sur le fait qu'un masque ne peut "vivre " que durant une seule saison.

6. On se demande effectivement quel argument ecclésiastique pourrait justifier que cette procession populaire soit considérée profane devant l'effigie de Saint Jean Baptiste alors qu'elle est maintenant tolérée devant celle de Saint Pierre... La démarche s'explique plutôt par une volonté d'ouverture de certains membres de l'Eglise tout en ménageant les autres. Aussi, Saint Pierre est logé dans une simple chapelle tandis que Saint Jean Baptiste patronne une Eglise qui jouxte les bâtiments paroissiaux. 